\title{
Les deux livres de l'ethnographe. Ethnologie et littérature en France entre 1930 et 1955
}

\section{Vincent Debaene}

\section{(2) OpenEdition}

12 Journals

Édition électronique

URL : http://journals.openedition.org/recherchestravaux/579

DOI : 10.4000/recherchestravaux.579

ISSN : 1969-6434

Éditeur

UGA Éditions/Université Grenoble Alpes

\section{Édition imprimée}

Date de publication : 15 mai 2013

Pagination : $39-51$

ISBN : 978-2-84310-248-6

ISSN : 0151-1874

Référence électronique

Vincent Debaene, "Les deux livres de l'ethnographe. Ethnologie et littérature en France entre 1930 et 1955 », Recherches \& Travaux [En ligne], 82 | 2013, mis en ligne le 15 novembre 2014, consulté le 08 septembre 2020. URL : http://journals.openedition.org/recherchestravaux/579; DOI : https://doi.org/ 10.4000/recherchestravaux.579 
Vincent Debaene

Columbia University, New York

\section{Les deux livres de l'ethnographe Ethnologie et littérature en France entre 1930 et 1955}

La grande difficulté posée par l'étude des "rapports» entre ethnologie et littérature tient au caractère extrêmement insaisissable de ces deux notions. Parle-t-on de corpus, de types de discours, de pratiques d'écriture, de disciplines...? Cette difficulté théorique se double d'une difficulté historique et axiologique : ce qui était considéré comme de la littérature autrefois, ce qui fut reçu comme de l'ethnologie hier, ne l'est plus forcément aujourd'hui. On est immédiatement renvoyé à la question des critères : «est-ce vraiment de la littérature?», "est-ce vraiment de l'anthropologie?». Et ces deux interrogations ne sont symétriques qu'en apparence puisque la première engage un jugement de valeur lorsque la seconde fait référence à un état du savoir à un moment donné. Considérer les rapports entre ethnologie et littérature suppose donc que l'on se défasse non seulement de toute conception implicite de l'anthropologie ou de la littérature qui informerait subrepticement l'analyse, mais aussi que l'on se débarrasse de tout finalisme, finalisme des histoires littéraires comme des histoires des sciences. Au fond, il s'agit simplement d'appliquer la leçon de l'ethnologue : plutôt que de prendre le risque d'importer des catégories et de les greffer artificiellement sur une réalité qui se dérobe, il vaut mieux d'abord regarder ce que les hommes font.

Pour cette raison, nous partirons d'un phénomène précis et isolé, qui constitue une sorte de curiosité bibliographique, et que l'on pourrait appeler les «deux livres» de l'ethnographe. Depuis les années I930, en effet, on observe que presque tous les ethnographes français partis sur leur terrain écrivent, à leur retour, non seulement une étude consacrée à la population auprès de 
laquelle ils ont séjourné, mais aussi souvent un «deuxième livre», un ouvrage plus «littéraire», en tout cas qui ne respecte pas la forme canonique de la monographie savante. Cet ouvrage est, qui plus est, publié non plus dans une revue ou une collection spécialisée, mais chez un éditeur généraliste : Gallimard, Plon ou Grasset ${ }^{\mathrm{I}}$.

Les cas les plus célèbres sont ceux de Michel Leiris qui écrit, d'une part, La langue secrète des Dogons de Sanga (Institut d'ethnologie, I9482) et, d'autre part, L’Afrique fantôme (Gallimard, 1934), et de Claude Lévi-Strauss qui, d'un côté, publie La vie familiale et sociale des Indiens nambikwara (Société des américanistes, 1948) et, de l'autre, Tristes tropiques (Plon, 1955). Ce ne sont cependant pas les seuls, et l'on trouve également ce «face-à-face» entre science et littérature chez Marcel Griaule par exemple qui, après avoir consacré deux ouvrages aux "divertissements abyssins", relate sa première mission en Éthiopie dans Les Flambeurs d'hommes (Calmann-Lévy, 1934) ou chez Alfred Métraux qui, un an à peine après la publication de sa volumineuse Ethnology of Easter Island, donne à Gallimard, sous le titre Lî̀le de Pâques, le récit de son enquête, récit que Bataille considérait comme «un des chefs-d'œuvre de la littérature française présente» qui «laisse loin derrière la masse de ces romans que le public reçoit sous le nom de littérature $»^{3}$. On pourrait encore ajouter les exemples de Maurice Leenhardt (Gens de la Grande Terre, Gallimard, 1937), de Jacques Soustelle (Mexique, terre indienne, Grasset, 1936), de Jehan Vellard (Une civilisation du miel, Gallimard, 1939), ou de Paul-Émile Victor (Boréal et Banquise, Grasset, 1938 et 1939).

Remarquable en tant que tel, ce schéma du double livre est d'autant plus étonnant qu'il apparaît précisément au moment où les ethnographes affirment avec emphase qu'ils ne sont pas des écrivains ou des "littérateurs", que l'étude de l'homme a désormais rompu avec la littérature et qu'elle est entrée dans l'âge scientifique : la nouvelle discipline a coupé les ponts avec le récit de voyage et les facilités de l'exotisme pour se fonder sur des documents et des faits bien établis, sans souci de la "couleur locale» et sans désir de plaire

I. Une première version de cet article a paru, sous le titre «L'ethnologue et l'écrivain au musée d'Ethnographie : chassés-croisés (1925-1955)", dans le recueil Panthéons scientifiques et littéraires : XIXe-XXe siècles (Arras, Artois Presses Université, 20I2). Je remercie les directeurs de cette publication, A.-G. Robineau-Weber, É. Thoizet et N. Wanlin, d'avoir accepté que ce texte soit ici partiellement repris. Il s'agit d'une présentation extrêmement résumée de l'argument du livre L'adieu au voyage. L'ethnologie française entre science et littérature, Gallimard, coll. "Bibliothèque des sciences humaines", 20Io. Cet ouvrage traite des rapports compliqués de l'ethnologie française à la littérature en même temps que de ceux - non moins compliqués de la littérature française à l'ethnologie.

2. Il s'agit en réalité du mémoire de fin d'études de Leiris à l'Institut d'ethnologie, soutenu dix ans auparavant, en 1938.

3. G. Bataille, «Un livre humain, un grand livre», Critique, n I05, février 1956, p. I00. 
au public. On notera également que les paires constituées par les deux livres de l'ethnographe ne sont pas superposables entre elles : la relation entre les travaux de Leiris sur la possession chez les Éthiopiens de Gondar et L'Afrique fantôme ne recouvre pas la relation entre Ethnology of Easter Island et Lîle de Pâques de Métraux, ou - pour prendre un exemple abondamment étudié dans l'Université américaine - entre Les Argonautes du Pacifique occidental de Bronisław Malinowski et son journal publié par sa veuve vingt-cinq ans après sa mort sous le titre A Diary in the Strict Sense of the Term. C'est un point important, car cela évite que la distribution entre les deux livres soit immédiatement repliée sur des partages institués entre "objectif» et «subjectif», entre "explication" et «interprétation", entre "froide raison" et "culture du goût», entre "écriture contrainte» (du savant) et "écriture souveraine» (de l'écrivain), ou entre «rigueur et progrès» (des sciences) d'un côté et «impressionnisme et traditionalisme» (des lettres) de l'autre.

Une question s'impose donc : comment comprendre ce paradoxe d'un recours à la «littérature" au moment même où l'on prétend s'en débarrasser pour accéder à la science? Après un bref rappel historique, nous envisagerons les contradictions épistémologiques de l'ethnologie de la période pour essayer de saisir ce qui motive un tel désir de "littérature» chez des savants pourtant si soucieux de rigueur et d'objectivité.

La fondation institutionnelle de l'ethnologie en France peut être circonscrite par deux dates - 1925 et 1938 - qui renvoient aux deux institutions centrales de la discipline. La première correspond à la création de l'Institut d'ethnologie de Paris qui se charge de la formation des ethnographes, la seconde à l'inauguration du musée de l'Homme, chargé quant à lui de la visibilité de cette nouvelle science et de la diffusion de ses «résultats». Cette inauguration est l'aboutissement d'un processus commencé en 1929 lorsque l'ancien musée d'Ethnographie du Trocadéro (qui datait lui-même de 1878) change de statut et passe sous la coupe du Muséum national d'histoire naturelle. Il est alors réorganisé selon des principes scientifiques, conformément aux exigences de la muséologie moderne. En 1934, le palais du Trocadéro est finalement démoli; il est remplacé par le palais de Chaillot pour l'Exposition universelle de 1937, et le musée d'Ethnographie laisse sa place au musée de l'Homme4.

Cette petite quinzaine d'années constitue une période capitale dans l'histoire de l'ethnologie française; c'est l'époque du compagnonnage avec l'avantgarde - certes mythifiée par la remémoration, mais opérante dans les années qui

4. Sur cette période, voir les travaux fondateurs de J. Jamin et l'ouvrage plus récent de Chr. Laurière, Paul Rivet, le savant et le politique, Paris, Éd. scientifiques du Muséum national d'histoire naturelle, coll. "Archives», 2008. 
suivent et jusqu'à aujourd'hui pour cette raison même - et d'un bouillant mélange entre des professeurs venus du Muséum ou de l'École des hautes études et des surréalistes dissidents et autres adeptes de l' «art moderne». Les uns et les autres se rencontrent autour de quelques lieux et de quelques institutions : le musée d'Ethnographie, donc, mais aussi la galerie d'art de Georges Wildenstein, rue du Faubourg-Saint-Honoré, une collection comme «L'Espèce humaine» chez Gallimard, la revue Arts et métiers graphiques et plus encore la revue Documents, animée par Georges Bataille et Michel Leiris. Dans le même temps, à l'Institut d'ethnologie, la première génération d'ethnographes est formée autour d'un impératif : l'enquête de terrain sur place, auprès des «indigènes». Les fondateurs de l'Institut d'ethnologie (Lucien Lévy-Bruhl, Marcel Mauss, Paul Rivet) sont des professeurs qui, à l'exception de Rivet, n'ont pas eux-mêmes connu d'expérience de terrain, mais ils vont l'exiger de leurs étudiants, transformant de ce fait le terrain en rite de passage et véritable consécration professionnelle. De là, pour la première génération formée dans les années 1930, le sentiment très puissant d'introduire une rupture (en particulier par rapport à l'ancienne ethnographie coloniale) et d'inaugurer une discipline. C'est qu'il ne s'agit plus seulement d'aller sur place et de collecter des pièces et des documents pour les envoyer en métropole; ce doit être la même personne qui voyage, qui collecte et qui interprète. On sort donc de la traditionnelle division du travail entre l'observateur - qui collecte - et le théoricien - qui compare et synthétise.

Si l'on considère les pratiques de voyage, l'ennemi de l'ethnographe, ce sera donc le touriste ou le voyageur amateur, celui qui ne possède pas les techniques d'observation et de collecte. Mais si l'on considère les écrits produits au retour, l'ennemi, ce sera le littérateur, celui qui n'a pas de méthode et qui se laisse aller aux effets de style, à la couleur locale, à l'impressionnisme. «Les effets artistiques doivent être l'objet de la plus grande méfiance», recommande par exemple Griaule dans les années 1940 dans ses cours de «méthode ethnographique» à la Sorbonnes. À l'inverse des littérateurs, les ethnologues, eux, sont des professionnels et des spécialistes; ils appliquent une méthode, ils ne se laissent pas abuser par les aspects les plus spectaculaires d'une société, ils refusent le pittoresque et revendiquent la monotonie de la forme.

Ce serait singulièrement simplifier le problème que d'essayer de comprendre l'âme de ce grand peuple autrement qu'au travers d'enquêtes superficielles de littérateurs ou de dilettantes. Il serait grand temps qu'on prenne au sérieux les faits sociaux où se révèle cette âme, et qu'on leur fasse l'honneur de les étudier comme une réaction chimique ou un problème de résistance des matériaux ${ }^{6}$.

5. M. Griaule, Méthode d'ethnographie, Paris, Presses universitaires de France, 1957, p. 83.

6. M. Griaule, "Une mission ethnographique et linguistique en Éthiopie (I928-1929)", L’Afrique française, août 1930. 
Qu'on n'attende ici ni littérature, ni habileté de reportage. Il ne m’importe que de montrer quasi nu le document naissant de l'observation directe d'une cérémonie, compte non tenu des innombrables informations recueillies en dehors d'elle .

On voit ici que la «naissance» de l'ethnologie obéit à un schéma qui ne lui est pas propre et qu'on retrouve lors de la constitution de toute science en France depuis le début du $\mathrm{XIX}^{\mathrm{e}}$ siècle : une science s'arrache à la littérature; une discipline naît lorsqu'elle cesse d'être de la littérature. En histoire naturelle, c'est ce schéma qui est au cœur de la rupture entre Cuvier et Buffon; et c'est ce schéma qu'on retrouve encore en histoire dans les années I870, dans le rapport des savants de la Revue historique à Michelet ou Guizot. De là, chez les savants français, un rapport compliqué, presque œedipien, à la littérature : la littérature est à la fois le contraire et le passé de la science; c'est à la fois ce dont on se coupe et ce dont on hérite. Les relations entre science et littérature (entre sciences et littératures, faudrait-il dire) sont instables et compliquées au cours des $\mathrm{XIX}^{\mathrm{e}}$ et $\mathrm{XX}^{\mathrm{e}}$ siècles; elles comportent plusieurs dimensions - sociologiques, politiques, culturelles... -, et elles varient à la fois dans le temps et selon les objets, mais théoriquement et $d u$ point de vue du savant qui innove, le modèle demeure inchangé : le rapport à la littérature est un rapport d'émancipation; il s'agit toujours de circonscrire un territoire au sein de la connaissance généraliste de l'homme de lettres, et de le soustraire aux discours qui ne satisfont pas aux nouveaux critères de scientificité. Et ces critères, au nombre de quatre, sont eux-mêmes invariables et indifférents à l'objet : privilège accordé à l'observation (contre l'imagination); souci de la méthode (contre les fantaisies et les combinaisons de l'esprit) ; monotonie assumée de la forme (contre l'éloquence et le style); délimitation d'un domaine d'experts (contre les amateurs et les "gens du monde») ${ }^{8}$.

On comprend dès lors que le passage du musée d'Ethnographie sous la coupe du Muséum d'histoire naturelle en 1929 soit vécu par les pères fondateurs de la discipline comme la grande victoire de l'ethnologie; c'est l'indice de l'accession à la science; cela signale la fin du règne des voyageurs mondains et des récits exotiques. De là, une dimension anti-littéraire très revendiquée dans les premières années de l'ethnologie : on passe de l'amateurisme esthète et artiste au professionnalisme du savant (même si dans les faits, les préoccupations esthétiques continuent à jouer un rôle central à la fois dans les pratiques de collecte et dans le mode d'exposition des objets au palais de Chaillot ${ }^{9}$ ).

7. M. Griaule, «Le chasseur du 20 octobre», Minotaure, $\mathrm{n}^{\circ}$ 2, 1934, p. 3 I.

8. Voir sur ce point, L'adieu au voyage..., ouvr. cité, p. 28-38.

9. Voir B. de l'Estoile, Le goût des autres. De l'Exposition coloniale aux Arts premiers, Paris, Flammarion, 2007, p. 185. 
Comme on le voit dans les passages cités de Griaule, le maitre-mot de ce changement, c'est le document : que l'ethnologie se fonde à présent sur des documents bien établis est le signe de son entrée dans la scientificité. Au fond, c'est cette exaltation du document et ce rejet de la littérature qui font se rejoindre les avant-gardes postsurréalistes et les ethnologues. Ce sont eux qui vont réunir Georges Bataille et Marcel Griaule au sommaire de la revue Documents. Le point de convergence entre l'ethnologie et l'avant-garde, ce n'est pas tant l'art nègre ou le "primitivisme» que la haine de la figure.

La difficulté, c'est que, en 1930, ce modèle documentaire, d'inspiration "positiviste» et qui place le musée en son centre est anachronique et qu'il ne convient pas à l'objet de l'ethnologie. L'une des ruptures fondamentales introduites par Durkheim et reprise ensuite par Mauss est la définition du fait social comme un fait moral, comme un fait mental. Au-delà des productions matérielles, au-delà des institutions et des règles de droit, ce qui caractérise une société dans sa singularité, ce sont certaines "façons de penser", certaines "façons de sentir", un "climat moral», une «mentalité» :

[...] pour penser et pour agir comme [les indigènes] font, pour avoir les institutions que nous constatons chez eux, il faut que leur esprit ne soit pas orienté comme le nôtre, que les cadres et le contenu de leur expérience ne coïncident pas tout à fait avec les nôtres [...]. Par conséquent, nous devons nous imposer un effort très pénible pour entrer dans leurs façons de penser et de sentir ${ }^{10}$.

L'entrée de l'ethnologie à l'Université via l'Institut d'ethnologie de Paris va ainsi de pair avec une mentalisation de son objet : une société, ce n'est pas seulement des masques et des outils, ni même des légendes et des contes; c'est d'abord un ensemble de dispositions affectives, une façon de se rapporter au monde. "Nous n'étudions plus seulement des choses tenues dans la main, ou visibles, explique Mauss dans ses cours d'ethnographie, mais des états de conscience ${ }^{\mathrm{II}}$.» Plus loin, il évoque la nécessité de saisir l'«atmosphère» d'une société :

Au terme de pareille enquête [l'étude de «la morale d'une société» à travers «sa littérature et plus spécialement ses proverbes»], on pourra définir la tonalité morale de la société observée, en s'efforçant de rester dans l'atmosphère de cette société : il est bien d'exercer la vendetta, il est bien de pouvoir offrir une tête humaine à sa fiancée ${ }^{12}$.

Avec le document, l'atmosphère est l'autre grand mot de la période. Mauss écrit par exemple à la fin de l'Essai sur le don: "Une partie considérable de

Io. L. Lévy-Bruhl, "Une heure avec... Entretien avec Fr. Lefevre», Les Nouvelles littéraires, février 1927.

II. M. Mauss, Manuel d'ethnographie, Paris, Payot, 1967, p. I09.

I2. Ibid., p. 2 OI. 
notre morale et de notre vie elle-même stationne toujours dans cette même atmosphère du don, de l'obligation et de la liberté mêlées ${ }^{13}$." En histoire des mentalités à la même époque, on parle d' "atmosphère mentale». C'est que, en dépit - ou en raison - de son caractère diffus et évanescent, l'atmosphère est la métaphore tout indiquée pour dire cette circulation diffuse des « façons de sentir» et la possibilité pour le savant de s'en «imprégner».

On voit en quoi cette caractérisation du fait social comme "atmosphère", «climat moral» ou «tonalité morale» entre en conflit avec le modèle muséal et documentaire qui inspire Rivet au moment où il obtient que le musée d'Ethnographie du Trocadéro passe sous la coupe du Muséum d'histoire naturelle. Comment faire pour restituer un objet qui précisément ne se collecte pas? Les ethnologues sont ainsi constamment pris entre deux postulations : d'un côté, au nom de l'objectivité et contre le pittoresque, ils revendiquent le caractère strictement documentaire de leurs travaux et n'oublient jamais de renvoyer à l'inventaire monographique et aux collections du musée; de l'autre, ils ne cessent de déplorer l'insuffisance de la pièce et son incapacité à restituer l' "atmosphère» de la société étudiée.

Cette manière de rassembler des faits humains concrets [la collecte] est maintenant désuète et ne se suffit plus à elle-même. [...] L'objet n'est en effet qu'une phase très restreinte de vastes activités, et c'est une piètre victoire que la récolte de ces témoins muets s'ils ne peuvent être remis, grâce à une documentation intensive, dans l'atmosphère de la société qui les a produits ${ }^{14}$.

Dans les années 1930, on trouve ainsi de nombreux témoignages qui disent l'insatisfaction des ethnologues devant une science des sociétés réduite à la collecte de documents et de bois morts, réduction qui leur parait à la fois trahir leur objet et leur expérience de terrain :

Un à un les objets sont affublés d'un petit numéro et repérés sur le plan. Finie la liberté! Le document est bien englouti, engrené dans la machine à raisonner, valorisé, curieux, exploitable, surgi à la lumière, perdu à jamais pour les morts qui l'avaient laissé là ${ }^{15}$.

J'avais à étudier les épaves d'un grand naufrage, [un] pénible assemblage de documents disparates et médiocres. [...] [Le] changement [est] si profond qu'il apparait presque impossible de reconstituer l'atmosphère sociale et morale dans laquelle vivait un indigène il y a quatre-vingts an $s^{16}$.

I3. M. Mauss, Sociologie et anthropologie, Paris, Presses universitaires de France, coll. "Quadrige», I950, p. 258.

I4. M. Griaule, Méthode de l'ethnographie, ouvr. cité, p. 29.

I5. M. Griaule, Les Sâ̂ légendaires, Paris, Gallimard, 1943, p. 37.

I6. A. Métraux, Lî̀le de Pâques, Paris, Gallimard, coll. "L'Espèce humaine», I94I, p. IO; p. 88. 
De là, toute une rêverie des ethnologues autour de l'idée de document humain, de document vivant, ou de document évocateur : comment faire pour qu'un document reste humain? Comment faire pour qu'il conserve quelque chose de cette atmosphère dans laquelle il était baigné à l'origine?

Il y a là une contradiction très profonde dont on trouve trace dans les méthodes et les manuels. En ouverture du Manuel d'ethnographie, Mauss insiste par exemple sur l'exactitude qui doit être le souci majeur de l'ethnographe, dont la tâche première est d'"enregistrer des faits"; il qualifie ainsi la "science ethnologique» de "science de constatations et de statistique». Pourtant, il poursuit par ces mots : «La sociologie et l'ethnologie descriptive exigent qu'on soit à la fois chartiste, historien, statisticien... et aussi romancier, capable d'évoquer une société tout entière ${ }^{17}$.» Cet ajout, ou plutôt ce remords, est caractéristique des premières années de l'ethnologie : il montre que malgré les revendications de science et le souci de ne produire qu'un enregistrement des faits, la restitution de l'atmosphère, du climat moral, de la tonalité morale requiert un travail de la langue - et même au sens strict, un travail rhétorique puisqu'il s'agit de "faire sentir les façons de sentir». Voici par exemple ce qu'écrit Griaule dans sa Méthode de l'ethnographie :

Il est [...] indispensable de présenter au lecteur les faits dans leurs détails mêmes [...] l'écrivain ne s'interposant que pour un minimum d'élaboration. Inversement, du point de vue de l'atmosphère dans laquelle se sont déroulés les faits, l'écrivain a le loisir d'introduire des impressions, des incidents subjectifs qui seront des plus utiles. C'est qu'en effet, s'il s'agit d'un rite, par exemple, cette activité toujours spectaculaire comporte une gesticulation et une formulation obéissant à des règles esthétiques diffuses, provoquant des réactions esthétiques précises. Il ne serait pas exact de reproduire, même très fidèlement, cette gesticulation et cette formulation sans leur rendre par des moyens également esthétiques leur aspect réel. Cette forme de description d'une société s'adressera donc à la sensibilité du lecteur autant qu'à son intelligence ${ }^{18}$.

Il est donc des faits pour lesquels la "reproduction", "même très fidèle", n'est tout bonnement pas "exacte»; dans le cas du rite, des «règles esthétiques» suscitent des "réactions esthétiques» qui exigent des "moyens esthétiques».

On se trouve donc placé devant deux nécessités contradictoires. L'écrivain doit disparaître quand il s'agit d'exposer la marche d'un rite, et d'autre part, il devra faire appel à toutes les ressources de sa personnalité pour rendre l'atmosphère du rite. Dans le premier cas, il emploiera un style froid, voire plat; il sacrifiera tout effet littéraire à la précision. Il devrait s'inspirer du style du Code civil ou de l'article d'encyclopédie [...]. Dans le second cas, il devra être bon littérateur' ${ }^{19}$.

I7. M. Mauss, Manuel d'ethnographie, ouvr. cité, p. 8.

I8. M. Griaule, Méthode de l'ethnographie, ouvr. cité, p. I03.

19. Ibid., p. IO3-IO4. 
Le même Griaule qui, en 1930, rejetait les «enquêtes superficielles de littérateurs ou de dilettantes" reconnaît ici que l'ethnographe doit parfois se montrer «bon littérateur».

Cette injonction contradictoire - le document et l'évocation - est la première explication du «deuxième livre» de l'ethnologue : dans la plupart des cas (au moins dans les années 1930), ce deuxième livre est écrit pour compenser le travail mortifère de la science, pour restituer l'"atmosphère» de la société étudiée. Voici ce que confie Métraux à Leiris alors qu'il prépare pour la collection "L'Espèce humaine» son très bel ouvrage intitulé Lîle de Pâques:

C'est-à-dire que l'ethnographe [...] doit étudier sa tribu comme le ferait un romancier et la faire vivre en usant des points de vue et des procédés du roman [...]. Ne pas aller disséquer des hommes, mais étudier leur vie, donner d'une tribu l'image de sa vie et ne pas disséquer son corps. Présenter les rouages fonctionnant et non pas démontés et classés suivant des règles parfaitement arbitraires et empiriques. Voyez pour cela le cours de Mauss. Non, ce que je veux c'est garder toute cette cuisine pour moi, mais donner aux autres la sensation de vie que doit produire le contact avec des hommes ${ }^{20}$.

Les écrits des ethnographes des années 1930 - non certes leurs écrits scientifiques, mais leurs correspondances, leurs journaux de terrain, etc. - portent fréquemment la trace d'un questionnement proprement rhétorique : comment faire sentir les façons de sentir? Quelle figure de style sera la mieux à même de traduire une atmosphère? On connaît par exemple la célèbre description du coucher de soleil au début de Tristes tropiques. Or ce «morceau» - écrit en mars 1938 sur le pont du bateau qui emmène Lévi-Strauss au Brésil et qui sera repris dix-sept ans plus tard dans Tristes tropiques - était au départ conçu comme un exercice de style, au sens strict, presque scolaire, du terme. Voici comment Lévi-Strauss le présente :

$\mathrm{Si}$ je trouvais un langage pour fixer ces apparences à la fois instables et rebelles à tout effort de description, s'il m'était donné de communiquer à d'autres les phases et les articulations d'un événement pourtant unique et qui jamais ne se reproduirait dans les mêmes termes, alors, me semblait-il, j'aurais d'un seul coup atteint aux arcanes de mon métier : il n'y aurait pas d'expérience bizarre ou particulière à quoi l'enquête ethnographique dût m'exposer, et dont je ne puisse un jour faire saisir le sens et la portée $e^{21}$.

20. Lettre d'Alfred Métraux à Michel Leiris, envoyée d'Honolulu le 6 mai 1936, citée par G. Poitry, "Carrefour des poètes : Michel Leiris et Alfred Métraux», Bulletin du Centre genevois d'anthropologie, $\mathrm{n}^{\circ}$ 5, 1995-1996, p. 7 .

2I. Cl. Lévi-Strauss, Tristes tropiques [1955], dans CEuvres, Paris, Gallimard, coll. «Bibliothèque de la Pléiade», 2008, p. 50-51. 
L'ouvrage le plus révélateur - et le plus réussi - correspondant à une telle tentative de restitution de l'atmosphère est sans aucun doute L'̂̀le de Pâques ${ }^{22}$ de Métraux, qui se donne comme une tentative très nostalgique d'évocation - au sens strict et littéral - d'une société disparue à partir de documents le plus souvent mutilés (statues, tablettes, outils, mythes, etc.). Au moment de donner au lecteur les textes des légendes des anciens Pascuans (par ailleurs intégralement restitués et littéralement traduits dans sa monographie scientifique, Ethnology of Easter Island), Métraux précise ainsi :

Je voudrais, dans ces pages écrites en français, faire passer un peu de la saveur naïve du texte original, tout en supprimant les longueurs et les répétitions. Que le lecteur veuille bien s'imaginer de grands champs de pierre, un rivage charbonneux, des collines verdoyantes dans le lointain, et la mer, sur laquelle s'étalent comme des plaques de bronze, la mer, cette vieille amie des Polynésiens, dont les vagues et les vents apportèrent les premiers hommes sur le rivage où j'écoutais leur histoire ${ }^{23}$.

Dans cette tentative de restitution de l'atmosphère de la vieille société pascuane, Métraux fait flèche de tout bois et multiplie les dispositifs rhétoriques : ekphrasis, jeu sur les temps, "tableaux imaginaires", citations de littérature indigène... Il décrit par exemple une cérémonie de "salutations larmoyantes» telle qu'on aurait pu encore l'observer au XIX ${ }^{\mathrm{e}}$ siècle; il reconstitue, au style indirect libre, le «cycle de vie» des anciens Pascuans, et il consacre bien sûr plusieurs pages à l'érection des fameuses statues :

Il ne faudrait pas cependant projeter au milieu du Pacifique le tableau familier de la construction des pyramides d'Égypte. Les statues n'ont pas été mises en place par des grappes d'esclaves, mais par des hommes libres, heureux de participer à une entreprise menée pour la plus grande gloire de la tribu ou de la famille. C'est le tumulte joyeux des fêtes qu'il nous faut évoquer lorsque nous pensons à cet effort ${ }^{24}$.

"Il faut imaginer", «Il ne faudrait pas croire... mais plutôt se représenter»; "Que le lecteur veuille bien s'imaginer» sont des formules qui émaillent L’̂̀le de Pâques comme autant de refrains.

On observe ainsi un étonnant croisement historique puisque, pour justifier leurs tentatives d'évocation, les ethnologues vont promouvoir une conception instrumentale, pour ne pas dire archaïque, de la littérature, conception qui n’a rien à voir avec celle de l'avant-garde : à leurs yeux, la littérature redevient

22. Cet ouvrage connaît deux versions sensiblement différentes, bien que la date du copyright n'ait pas été modifiée lors des rééditions successives. La première version du texte date de 194I ; elle était accompagnée de photographies et était nettement plus «littéraire» que la seconde, datée de 195I, qui est celle qu'on trouve aujourd'hui dans le commerce.

23. A. Métraux, Lî̀le de Pâques, ouvr. cité, p. I80.

24. Ibid., p. I52. 
l'art du bien dire, la technique de représentation et de restitution des réalités morales. Autrement dit, la rencontre entre l'avant-garde et l'ethnologie dans les années 1930 relève d'un effet de chiasme très curieux. D'un côté, les ethnologues regardent du côté de la littérature en raison de sa capacité supposée à restituer l'atmosphère d'une société, en raison de sa puissance rhétorique et de son aptitude à faire sentir les façons de sentir. C'est ainsi que Griaule réclame de l'ethnographe qu'il soit «bon littérateur» ou qu'il invite Leiris à participer à la Mission Dakar-Djibouti au titre d' «homme de lettres» de la mission. Mais d'un autre côté, l'avant-garde surréaliste ou postsurréaliste regarde du côté de l'ethnologie au nom du document et de sa capacité à défaire l'espace de la représentation verbale ou muséale. C'est cela qui fait la fascination de Breton pour le document et l'intérêt de Bataille pour l'ethnologie : l'idée que le document "pris sur le vif", prélevé à même le vivant, permettra, par une sorte de contagion, de briser les cadres de l'expérience commune - qu'il permettra, par exemple, de restaurer, une indistinction primitive entre l'esthétique, l'érotique et l'économique. On est alors à cent lieues de la littérature comme art de l'éloquence et de la figure; il s'agit au contraire de sortir de la représentation. Mauss voudrait que l'ethnologue se fasse "romancier», mais les surréalistes détestent le roman, et lorsque Bataille convoque Griaule dans ses écrits sur l'art primitif, ce n'est certes pas le grandiloquent récit ethnographique Les Flambeurs d'hommes qu'il mentionne, mais bien le recueil savant Silhouettes et graffiti abyssins dont l'objet avait tout pour le séduire ${ }^{25}$.

Il resterait à considérer les autres formes de partage entre les deux livres de l'ethnologue. Toutes, en effet, ne correspondent pas à cette répartition des rôles par laquelle la littérature est appelée à compenser les insuffisances de la science. C'est le cas en particulier de L'Afrique fantôme et de Tristes tropiques qui ne proposent nullement une autre version - plus humaine, plus sensible, plus «littéraire», etc. - des enquêtes de terrain de leurs auteurs. Plutôt que de les comparer aux travaux scientifiques contemporains, il vaut mieux envisager ces deux ouvrages en les resituant dans la dynamique de production de l'œuvre scientifique. Malgré tout ce qui sépare Leiris de Lévi-Strauss

25. Il s'agit de dessins illicites tracés par les enfants sur les portes et les murs des églises du Godjam; dans ces œuvres doublement sacrilèges, par leur apparence et par le lieu qu'elles souillent, Bataille décèle un processus répétitif par lequel l'altération fait naître un nouvel objet qui est altéré à son tour, et ainsi de suite à travers une série continue de défigurations successives. La primitivité de cet art ne tient donc ni à son caractère enfantin, ni à son caractère éthiopien, mais à ce qu'il révèle la dépense originelle et l'«altération des formes présentées» antérieures à tout souci esthétique (voir «L'art primitif», Documents, nº 7, 1930, p. 389-397). 
au plan du tempérament, des principes théoriques comme de l'ampleur de l'œuvre (littéraire d'un côté, savante de l'autre), les schémas sont en effet similaires : d'abord, une enquête de terrain décevante, dont on revient avec un sentiment d'échec, ce qui n'empêche pas qu'elle donne lieu à un travail universitaire acceptable (La langue secrète des Dogons de Sanga pour Leiris; La vie familiale et sociale des Indiens nambikwara pour Lévi-Strauss); en même temps que cette enquête, une première tentative littéraire sous la forme d'un récit d'initiation manquée (L'Afrique fantôme pour Leiris; la pièce L'Apothéose d'Auguste pour Lévi-Strauss ${ }^{26}$ ); quinze ou vingt années plus tard, une seconde tentative littéraire - véritable expérience d'écriture cette fois, qui bouleverse radicalement les coordonnées du rapport entre le sujet et l'objet auquel il s'applique - (Biffures et Fourbis pour Leiris; Tristes tropiques pour LéviStrauss); enfin, un travail savant qui est en quelque sorte l'aboutissement de cette transformation subjective (La possession et ses aspects théâtraux chez les Éthiopiens de Gondar pour Leiris; La pensée sauvage et les Mythologiques pour Lévi-Strauss). Dans ces deux cas, le livre "littéraire» est le résultat d'une expérience d'écriture qui prolonge une enquête demeurée inachevée, le passage par l'écriture jouant un rôle effectif d'articulation entre expérience vécue et discours de savoir. Il permet de mener à son terme une expérience de terrain qui était demeurée inaccomplie, car entreprise sur des bases fallacieuses : «sortir de soi " pour Leiris; faire l'expérience d'une altérité pure et préservée pour Lévi-Strauss. Le «deuxième livre» est alors moins le deuxième que le premier, c'est-à-dire celui qui rend possible l'œuvre à venir - moins un supplément à l'œuvre savante que sa condition. Ainsi, la littérature qui en un sens avait été congédiée par l'évolution d'une discipline de plus en plus savante, de plus en plus autonome et hermétique, revient par la bande, non certes comme représentation, mais à travers les expériences que l'écriture permet et qui en font à son tour une sorte de terrain : non plus seulement un lieu, mais une épreuve sur soi et en soi.

À partir des années 1950, les relations entre anthropologie et littérature changeront radicalement de visage. Elles ne disparaîtront pas mais elles seront totalement reconfigurées sous l'influence de différentes évolutions : la décolonisation; l'essor du marxisme et du structuralisme dans les sciences sociales; une politisation de l'ethnologie; l'affirmation des voix indigènes,

26. Cette pièce, commencée par Lévi-Strauss en août I938, alors qu'il était encore sur son terrain, raconte sur un mode très «sartrien " l'échec d'un explorateur, Cinna, qui, de retour à Rome après des années de voyages, s'aperçoit que son existence aventureuse reposait sur un mensonge. Cinna figure de toute évidence une allégorie de l'ethnographe de retour à sa civilisation, obligé de reconnaître que son expérience de terrain ne lui a accordé aucune révélation. Voir Euvres, ouvr. cité, p. I632-1650 et la notice de Tristes tropiques, ibid., p. I699-1700. 
etc. De tels changements mériteraient bien sûr de longues études, mais ils montrent au moins une chose : il est peu fructueux de penser les échanges entre ethnologie et littérature en considérant l'une et l'autre comme de vastes entités dont on observe ensuite les chevauchements ou les croisements - ce qui se croise, ce ne sont jamais que des idées de l'ethnologie et des idées de la littérature. 